\title{
Alimentation et maîtrise de la qualité protéique et technologique des laits en élevage
}

\author{
O Colin, F Laurent, B Vignon \\ INRA-ENSAIA, laboratoire de sciences animales, 2, avenue de la Forêt-de-Haye, \\ BP 172, 54505 Vandouure-lès-Nancy cedex, France
}

(Reçu le 16 juin 1992; accepté le 5 avril 1993)

\begin{abstract}
Résumé - L'influence d'une modification du niveau des apports alimentaires sur les caractéristiques physicochimiques du lait (protéines en particulier) et sur le rendement fromager obtenu en usine a été étudiée dans 16 troupeaux sur des laits de mélange de 2 traites consécutives. Après ajustement des rations pour satisfaire aux recommandations alimentaires de l'INRA, un apport supplémentaire de $2,5 \mathrm{~kg}$ d'aliment concentré a été réalisé selon un schéma expérimental en inversion. Cet apport permet un gain de $0,6 \mathrm{~g} \%$ de taux protéique et de $27 \mathrm{~g}$ par vache et par jour de matière protéique $(P<0,01)$. La teneur en caséines totales augmente dans les mêmes proportions qle le taux protéique. Les proportions relatives des différentes caséines et protéines du lactosérum ne sont pas significativement influencées par le niveau d'apport alimentaire, hormis le pourcentage d'as-CN. L'apport supplémentaire d'aliment concentré accroît le rendement fromager seulement lorsqu'il est exprimé en matière sèche $(+1,4 \%)$.
\end{abstract}

apports alimentaires / protéines / rendement fromager / lait de vache

Summary - Effect of nutrient supply level on the quality of milk on farms. The effects of changes in nutrient supply level on the physico-chemical characteristics of milk (especially proteins) and on cheese yield in the dairy industry were measured in 16 herds for 2 consecutive milking bulks. After the diets were adjusted to INRA's recommended level, an additional $2.5 \mathrm{~kg}$ concentrate was supplied in a change-over design. This supply increased true milk protein content by $0.6 \mathrm{~g}$ per 1000 and protein yield by $27 \mathrm{~g}$ per cow $/ d(\mathrm{P}<0.01)$. Casein content increased in the same proportions to true protein content. The level of nutrient supply did not significantly influence the proportions of the various caseins and whey proteins except for the proportion of $\alpha S-C N$. An additional supply of concentrate only increased DM cheese yield $(+1.4 \%)$.

nutrient supply / true protein / cheese yield / bovine milk 


\section{INTRODUCTION}

La transformation fromagère constitue un atout important de la filière laitière française. Parmi les critères de composition physicochimique du lait, le taux protéique (TP) est le principal paramètre intervenant dans la détermination du rendement fromager (Mocquot et al, 1963 ; Maubois et al, 1970 ; Banks et al, 1984 ; Gilles et Lawrence, 1985 ; Colin et al, 1992). Les industriels laitiers mettent souvent en cause la baisse du taux protéique pour expliquer les chutes ou les anomalies de rendement.

L'influence de différents facteurs zootechniques sur les variations du taux protéique a fait l'objet de nombreux travaux (Journet et Rémond, 1980 ; Rogers et Stewart, 1982 ; Hoden et al, 1984 ; Rémond, 1985 ; Barillet et al, 1987 ; Rémond et Journet, 1987 ; Sutton, 1989). Parmi les facteurs nutritionnels, le rôle du niveau d'apport énergétique sur les teneurs des différentes protéines du lait, sur les paramètres technologiques et sur le rendement fromager a été particulièrement souligné par de nombreux auteurs ( $\mathrm{Ng}$ Kwai Hang et al, 1982 ; Grandison et al, 1984, 1985 ; Vertes et al, 1989 ; Vertes et Hoden, 1989; Garel et Coulon, 1990 ; revue d'Hoden et al, 1991 ; Laurent et al, 1992). La diversité des méthodes destinées à apprécier l'aptitude fromagère des laits rend difficile l'interprétation des différences de résultats entre essais. De plus, les expérimentations ont souvent été réalisées avec de faibles effectifs compte tenu du coût et de la complexité des analyses effectuées en laboratoire. Enfin, peu d'études ont mesuré le rendement fromager en usine sur des volumes importants de lait.

L'objectif de ce travail a été de préciser les améliorations de la qualité du lait pouvant être attendues d'une meilleure maîtrise de l'alimentation des troupeaux dans les conditions de la pratique. L'expérimentation a consisté à modifier dans des élevages le niveau d'apport énergétique pour en étudier les répercussions, d'une part, sur les caractéristiques physicochimiques des laits de troupeau, notamment les teneurs en protéines, et, d'autre part, sur le rendement fromager mesuré en usine.

\section{CONDITIONS EXPÉRIMENTALES}

\section{Caractéristiques des troupeaux}

L'essai a été réalisé dans 16 troupeaux laitiers du sud-ouest de la Meurthe-et-Moselle. Les animaux sont de race Prim Holstein, l'effectif moyen par troupeau est de $49( \pm 13)$ vaches laitières. Le niveau moyen de production par lactation des troupeaux est de $6871 \mathrm{~kg}( \pm 809)$ de lait. Le taux protéique moyen par lactation de $30,7 \mathrm{~g} / \mathrm{l}( \pm 0,5)$ est inférieur de $0,2 \mathrm{~g} / \mathrm{l}$ à celui des laits collectés sur la même zone. L'index taux moyen de matière utile est faible $(-0,40 \mathrm{~g} / \mathrm{kg}$ en moyenne) et négatif pour tous les élevages sauf un, alors que la moyenne de l'index quantité de lait est de $+299 \mathrm{~kg}( \pm 374)$. Quatre-ving-quatre pour cent des vêlages ont lieu d'août à décembre, ce qui porte le stade de lactation moyen en début d'essai à 16,3 $( \pm 2,8)$ semaines. A l'exception d'une exploitation, l'ensilage de maïs représente toujours plus de $50 \%$ de l'apport en matière sèche des fourrages de la ration hivernale. Les vaches laitières reçoivent en moyenne $1023 \mathrm{~kg}( \pm 183)$ de concentré par an (mesure effectuée par le contrôle laitier). Les effectifs de vaches en lactation sont stables tout au long de l'expérimentation.

\section{Schéma expérimental}

L'essai a comporté 4 périodes (2 périodes préexpérimentales et 2 périodes expérimentales) de 3 semaines chacune. Pendant la première période préexpérimentale, les animaux sont alimentés selon les pratiques initiales des éleveurs puis en période préexpérimentale 2 , les rations sont ajustées pour satisfaire aux recommandations alimentaires de I'INRA. Cet ajustement des 
rations aux recommandations a consisté en une distribution supplémentaire de fourrage (4 élevages), des apports de correcteur azoté (3 élevages), des apports de concentré énergétique (6 élevages) ou une modification complète du plan de complémentation (3 élevages).

Les exploitations sont ensuite réparties en 2 groupes de 8 élevages. En période expérimentale 1 , les apports alimentaires sont maintenus au niveau des recommandations de l'INRA pour le premier groupe alors qu'une distribution supplémentaire de $2,5 \mathrm{~kg}$ de concentré est effectuée pour les vaches du deuxième groupe. En période expérimentale 2, le schéma est inversé, les animaux du premier groupe recevant $2,5 \mathrm{~kg}$ de concentré supplémentaire alors que les animaux du deuxième groupe reçoivent de nouveau des apports correspondants aux recommandations alimentaires de I'INRA. L'aliment concentré distribué a eu des valeurs UFL $/ \mathrm{kg}$ brut comprises entre 0,93 et 1,00 et un rapport PDI/UFL variant entre 100 et 135 selon les élevages.

\section{Mesures de la production et de la composition du lait}

Afin de mesurer l'effet de l'apport supplémentaire de concentré, les mesures suivantes ont été effectuées sur les 16 troupeaux:

- production laitière, taux butyreux (TB) et protéique (TP) (analyseur IRMA) et dénombrement leucocytaire (appareil Fossomatic) pour chaque vache des 16 troupeaux sur 2 traites consécutives, une fois par semaine ;

- taux protéique et taux butyreux (analyseur IRMA), teneurs en azote total, azote non protéique après précipitation des protéines à l'acide trichloroacétique $(12 \%$ de la solution finale), azote soluble après précipitation des caséines à l'acide acétique (1N) par ajustement du pH à 4,6 (méthode de Kjeldahl, dosage automatique sur appareil Vapodest 6), nature et pourcentage des caséines et des protéines du lactosérum (PLS) par chromatographie FPLC et HPLC respectivement (méthode d'Andrews et al, 1985), taille micellaire (granulomètre laser Autosizer II), $\mathrm{pH}$, teneurs en $\mathrm{Ca}, \mathrm{Na}$ totaux par spectrophotométrie d'absorption atomique (longueur d'onde : 423 et $589 \mathrm{~nm}$, largeur de fente : 0,7 et $0,2 \mathrm{~nm}$ respectivement) et en $\mathrm{P}$ total par colorimétrie (spectro- photomètre à $660 \mathrm{~nm}$ ). Ces mesures ont été réalisées sur les laits de mélange de 2 traites consécutives pour chaque élevage (lait de troupeau) en troisième semaine de chaque période.

\section{Transformations fromagères}

Sur les 2 mélanges des laits de 2 traites consécutives de 8 troupeaux (groupe A et groupe B), des fabrications fromagères de type pâte molle (camembert) ont été réalisées à l'usine de Vézelise de la laiterie Saint-Hubert en troisième semaine de chaque période. Ces fabrications sont réalisées sur environ $1700 /$ de lait standardisé à $20 \%$ de matière grasse.

Un ajout systématique de chlorure de calcium est effectué, à raison de $12,7 \mathrm{~g}$ de Ca par 100 I de lait. Les laits sont ensemencés avec des levains mésophiles et emprésurés à une température de $36^{\circ} \mathrm{C}$ à raison de $50 \mathrm{ml}$ de présure (force $1 / 10000$ ) par $100 \mathrm{I}$ de lait.

Les rendements fromagers sont déterminés 1 et 10 jours après transformation $(J+1$ et $J+10)$ par pesée d'environ $10 \%$ des fromages fabriqués à partir du volume mis en œuvre.

\section{Analyse des données}

Les variations induites par l'apport supplémentaire de $2,5 \mathrm{~kg}$ de concentré sont estimées en comparant les moyennes des caractéristiques physicochimiques et technologiques des 2 niveaux de distribution du concentré. Du fait de l'inversion des traitements retenue pour cette séquence expérimentale, les résultats moyens sont indépendants des effets du stade de lactation et de la période. Une analyse de variance (procédure GLM, logiciel SAS) est réalisée pour tester les différences, les facteurs retenus dans le modèle étant le niveau d'apport de concentré, la période et l'exploitation.

\section{RÉSULTATS}

L'apport supplémentaire de 2,5 kg d'aliment concentré a augmenté la production laitière de façon non significative $(+0,4 \mathrm{~kg} / L \mathrm{j})$, le taux protéique $(+0,6 \mathrm{~g} / \mathrm{kg}, P<0,01)$ et la quantité de matière protéique $(+27 \mathrm{~g} / \mathrm{j}$, 
$P<0,01)$. Le taux butyreux a diminué non significativement $(-0,8 \mathrm{~g} / \mathrm{kg}$ ) (tableau $\mathrm{l}$ ).

Le pourcentage de caséines dans les protéines totales et les pourcentages relatifs des différentes caséines et protéines du lactosérum n'ont pas été significativement modifiés, hormis le pourcentage d' $\alpha S$ $\mathrm{CN}$ dont l'augmentation est statistiquement significative $(P<0,05)$ (tableau I).
Les teneurs en calcium et phosphore ont augmenté légèrement mais non significativement $(+0,03$ et $+0,06 \mathrm{~g} / \mathrm{l}$ respectivement), alors que la teneur en sodium est restée stable. Le $\mathrm{pH}$ et la taille micellaire n'ont pas varié.

Le rendement fromager frais, mesuré 1 ou 10 jours après transformation, a eu tendance à augmenter.

Tableau I. Influence de la distribution supplémentaire de $2,5 \mathrm{~kg}$ d'aliment concentré sur la production, la composition et la valeur technologique du lait.

\begin{tabular}{|c|c|c|c|c|}
\hline & $\begin{array}{l}\text { Recomman- } \\
\text { dations }\end{array}$ & $\begin{array}{l}\text { Distribution } \\
\text { supplémentaire } \\
\text { de } 2,5 \mathrm{~kg} \text { de } \\
\text { concentré }\end{array}$ & $\begin{array}{l}\text { Seuil de } \\
\text { signification }\end{array}$ & $\begin{array}{l}\text { CV (\%) } \\
\text { (résiduelle } \\
\text { du modèle) }\end{array}$ \\
\hline \multicolumn{5}{|c|}{ Moyenne des laits des troupeaux } \\
\hline Nombre & 15 & 15 & & \\
\hline Production laitière $(\mathrm{kg})$ & $24,5(2,9)$ & $24,9(3,2)$ & NS & 2,9 \\
\hline Taux butyreux $(\mathrm{g} / \mathrm{kg})$ & $38,4(2,3)$ & $37,6(2,6)$ & NS & 3,2 \\
\hline Taux protéique $(\mathrm{g} / \mathrm{kg})$ & $29,6(1,2)$ & $30,2(1,4)$ & $P<0,01$ & 1,2 \\
\hline Matière protéique $(\mathrm{g} / \mathrm{NL} / \mathrm{j})$ & $730(71)$ & $757(79)$ & $P<0,01$ & 3,1 \\
\hline$\%$ caséines/protéines & $86,6(2,9)$ & $87,1(2,2)$ & NS & 2,1 \\
\hline$\% \alpha \mathrm{S}-\mathrm{CN}$ & $45,4(1,1)$ & $46,3(1,9)$ & $P<0,05$ & 1,9 \\
\hline$\% \beta-\mathrm{CN}$ & $35,8(1,6)$ & $35,4(2,5)$ & NS & 4,3 \\
\hline$\% \mathrm{~K}-\mathrm{CN}$ & $18,8(1,2)$ & $18,2(1,2)$ & NS & 7,1 \\
\hline$\% \alpha-L a$ & $27,7(2,5)$ & $27,7(2,6)$ & NS & 4,0 \\
\hline$\% \beta-\operatorname{LgA}$ & $41,8(6,6)$ & $41,0(7,0)$ & NS & 2,5 \\
\hline$\% \beta$-LgB & $30,5(6,4)$ & $31,3(6,2)$ & NS & 4,9 \\
\hline Calcium (g/l) & $0,92(0,09)$ & $0,95(0,14)$ & NS & 6,8 \\
\hline Sodium $(g / l)$ & $0,40(0,03)$ & $0,40(0,03)$ & NS & 4,4 \\
\hline Phosphore $(g / l)$ & $1,02(0,14)$ & $1,08(0,14)$ & NS & 10,3 \\
\hline $\mathrm{pH}$ & $6,74(0,05)$ & $6,74(0,04)$ & NS & 0,6 \\
\hline Taille micellaire $(\mathrm{nm})$ & $191(7)$ & $189(8)$ & NS & 1,9 \\
\hline \multicolumn{5}{|l|}{ Fabrication fromagère } \\
\hline Nombre & 2 & 2 & & \\
\hline $\begin{array}{l}\text { Rendement frais } \mathrm{J}+1 \\
(\mathrm{~kg} / 100 \mathrm{l})\end{array}$ & 12,4 & 12,5 & & \\
\hline $\begin{array}{l}\text { Rendement frais } \mathrm{J}+10 \\
(\mathrm{~kg} / 100 \mathrm{l})\end{array}$ & 11,1 & 11,2 & & \\
\hline
\end{tabular}

Moyenne (écart type). 


\section{DISCUSSION}

Après apport de $2,5 \mathrm{~kg}$ de concentré supplémentaire, le taux protéique des laits de troupeau a augmenté de $0,6 \mathrm{~g} / \mathrm{kg}$, soit de $0,25 \mathrm{~g} / \mathrm{kg}$ par $\mathrm{kg}$ de concentré. L'augmentation de production laitière de $0,4 \mathrm{~kg}$ n'a pas été significative. La réponse en terme de taux protéique varie de $-0,4$ à $1,5 \mathrm{~g} / \mathrm{kg}$ entre les exploitations. Dans certains cas, il est possible que l'apport énergétique supplémentaire ne corresponde qu'à un ajustement aux besoins réels des animaux du fait que l'estimation de la couverture des besoins des troupeaux n'est effectuée que de manière approchée.

Pour des laits individuels, une réponse variant entre 0 et $0,9 \mathrm{~g}$ de TP par $\mathrm{kg}$ de concentré supplémentaire pour des rations à base d'ensilage de maïs et des apports proches des recommandations a été enregistrée par Chenais et Kerouanton (1988), Pflimlin et Morhain (1988), Laurent et Gardeur (1989). Cette variabilité découle sans doute en partie des différences de réponse de la production laitière, qui a varié de $-0,1$ à $+0,8 \mathrm{~kg}$ de lait par $\mathrm{kg}$ de concentré, et d'une substitution variable entre fourrage et concentré selon les exploitations.

Rémond (1985) estime que la réponse du TP aux apports énergétiques est plus importante lorsqu'on se situe en dessous des besoins qu'au-dessus, mais des résultats récents (Coulon et Rémond, 1991) ont montré que la réponse était linéaire pour des bilans énergétiques se situant entre -3 et +3 UFL. Malgré l'incertitude quant à l'estimation de la couverture des besoins dans cet essai, les bilans énergétiques moyens des troupeaux doivent se situer dans cette plage.

Si l'on admet un coefficient de substitution entre fourrage et concentré de 0,5 , l'apport supplémentaire de $2,5 \mathrm{~kg}$ d'aliment concentré représente au plus 1,2 UFL. L'augmentation de TP enregistrée sous l'effet de la supplémentation en concentré représenterait donc $0,5 \mathrm{~g} / \mathrm{UFL}$, valeur équivalente à celle observée sur un grand nombre d'expérimentations (Rémond, 1985). Coulon et Rémond (1991), à partir d'une synthèse de 66 essais, chiffrent l'augmentation du TP à $0,6 \mathrm{~g} / \mathrm{kg}$ par UFL supplémentaire pour les essais réalisés en milieu de lactation ou les essais de longue durée.

Les exploitations retenues dans ce travail avaient un niveau de production laitière supérieur de $800 \mathrm{~kg}$ à la moyenne des troupeaux du département adhérant au contrôle laitier. Malgré ce bon niveau technique, les rations initialement distribuées ne correspondaient pas aux recommandations alimentaires de I'INRA, souvent par défaut d'appréciation des quantités de fourrage ingérées par les animaux. Les variations de production et de composition du lait entre les périodes préexpérimentales 1 et 2 divergent entre les exploitations $(-1,8$ à $+1,4 \mathrm{~kg} /$ jour pour la production laitière, $-0,4$ à $+2,3 \mathrm{~g} / \mathrm{kg}$ pour le TP). En effet, l'ajustement des rations a résulté de modifications du niveau des apports énergétiques et/ou azotés et de leur nature (fourrage, concentré), dont l'importance a varié selon les élevages. De plus, compte tenu de la durée séparant les 2 mesures effectuées en périodes préexpérimentales 1 et 2 ( 3 semaines), le stade de lactation influe également sur les variations de production et de composition du lait. If ne semble donc pas possible d'attribuer les variations de production et de composition du lait observées lors de l'ajustement des rations aux recommandations de I'INRA à des variations d'apports énergétiques et/ou azotés.

Le pourcentage de caséines dans les protéines totales ne varie pas avec l'augmentation des apports alimentaires, ce qui correspond aux observations de Journet et Rémond (1980), Le Doré et al (1986) et Vertes et al (1989). Parmi les différentes 
caséines et protéines du lactosérum, seul le pourcentage d' $\alpha S-C N$ a augmenté significativement lors de la séquence «supplémentation». Nous avons également noté une variation significative mais inverse du pourcentage d' $\alpha \mathrm{S}-\mathrm{CN}$ pour des laits individuels lors d'une augmentation des apports de concentré de $3 \mathrm{~kg}$ (résultats non publiés). Vertes et al (1989) ont mesuré une augmentation significative du pourcentage de $\kappa$-CN pour des laits de petit mélange, en passant d'une ration médiocre à une ration de bonne qualité mais avec des effectifs d'animaux faibles. Laurent et al (1992) rapportent une augmentation de la proportion de $\kappa-\mathrm{CN}$ et une diminution de $\beta-C N$ sous l'effet d'une supplémentation de 2,3 kg de concentré. $\mathrm{Au}$ vu de ce faible nombre de résultats et de leur variabilité, il apparaît que l'incidence du niveau des apports alimentaires sur les proportions des différentes caséines reste à préciser.

Les rendements fromagers frais mesurés en industrie augmentent peu avec l'apport de $2,5 \mathrm{~kg}$ de concentré $(+0,5 \%$ à $\mathrm{J}+10$ ) alors que le TP a augmenté de $2 \%$. Ces différences peuvent s'expliquer en partie par une variation de la teneur en MS des fromages fabriqués, respectivement $44,3 \%$ en séquence "recommandation" et $44,8 \%$ en séquence «supplémentation». Exprimé en quantité de MS (rendement frais $x$ teneur en MS), le rendement augmente de $1,4 \%$.

\section{CONCLUSION}

Les résultats acquis dans cette étude réalisée dans des élevages sur des laits de mélange confirment les résultats antérieurs obtenus sur des laits individuels quant à l'importance du niveau des apports alimentaires, et principalement du niveau d'apport énergétique pour améliorer le taux protéique du lait. Dans cette expéri- mentation, la distribution de $2,5 \mathrm{~kg}$ de concentré supplémentaire après ajustement des rations pour satisfaire les recommandations alimentaires de I'INRA améliore le TP de $0,6 \mathrm{~g} / \mathrm{kg}$ et la quantité de protéines de $27 \mathrm{~g}$ par vache et par jour. L'augmentation de la quantité de concentré distribué entraînant une diminution de fourrage ingéré, l'intérêt économique d'une telle supplémentation dépend principalement du coût respectif de I'UF du fourrage et de l'aliment concentré. Pour $1 \mathrm{I}$ de lait payé $2 \mathrm{~F}$ et $1 \mathrm{~g}$ de TP 3 centimes, le supplément de produit pour un niveau de production de 25 I se situe autour de 1,20 F par vache. Il serait prudent de considérer également les changements induits par de telles pratiques sur le système d'exploitation, et notamment la répartition des surfaces entre les différentes cultures.

Dans les élevages où cet essai a été conduit, qui sont d'un bon niveau technique, l'ajustement strict des rations aux recommandations a permis d'améliorer les performances de production et de composition du lait d'une partie des troupeaux. Dans ces exploitations, la pesée périodique des fourrages distribués permettrait de mieux adapter la nature et la quantité des concentrés distribués et ainsi d'optimiser les performances des animaux. Plus généralement, dans les élevages où l'alimentation est moins bien maîtrisée, l'ajustement des rations aux recommandations devrait conduire à une amélioration des résultats de plus forte amplitude, tout au moins pour les quantités de protéines produites, par augmentation soit de la production laitière, soit du TP.

Les proportions relatives des caséines dans les protéines totales et des différentes caséines ou protéines du lactosérum ne varient pas ou peu avec le niveau d'apport énergétique. Le taux protéique semble donc suffisant pour apprécier l'aptitude fromagère des laits lorsque les essais portent sur le niveau des apports alimentaires. 
Cependant, les rendements fromagers exprimés en matière brute ne sont que très légèrement améliorés ; le rendement fromager exprimé en matière sèche augmente de manière plus importante après la distribution du concentré supplémentaire.

\section{REMERCIEMENTS}

Nous tenons à remercier pour leur soutien financier l'Association nationale de la recherche technique, la Direction générale de l'alimentation du ministère de l'Agriculture, les industriels laitiers réunis au sein de l'association ARILEST, et par." ticulièrement l'Union lorraine des producteurs de lait et la région Lorraine.

\section{RÉFÉRENCES}

Andrews AT, Taylor MD, Owen AJ (1985) Rapid analysis of bovine milk proteins by fast protein liquid chromatography. $J$ Chromatogr 348, 177-185

Banks JM, Muir DD, Tamine AY (1984) Equations for estimation of the efficiency of Cheddar cheese production. Dairy ind int 49, 1417

Barillet F, Bonaiti B, Boichard D (1987) Amélioration génétique de la composition du lait des brebis, des chèvres et des vaches. In : Le lait, matière première de lindustrie laitière. INRA Publications, CNRA, route de SaintCyr, 78000 Versailles, 129-149

Chenais F, Kerouanton J (1988) Ensilage de maïs pour vaches laitières: quel concentré choisir? In : CR 88062. ITEB, 149, rue de Bercy, 75595 Paris Cedex 12

Colin O, Laurent F, Vignon B (1992) Variations du rendement fromager en pâte molle. Relations avec la composition du lait et les paramètres de la coagulation. Lait 72, 307-319

Coulon JB, Rémond B (1991) Variations in milk output and milk protein content in response to the level of energy supply to the dairy cow : a review. Livest Prod Sci 29, 31-47

Garel JP, Coulon JB (1990) Effet de l'alimentation et de la race des vaches sur la fabrica- tion de fromage d'Auvergne de SaintNectaire. INRA Prod Anim 3, 127-136

Gilles J, Lawrence RC (1985) The yield of cheese. NZ J Dairy Sci Technol 20, 205-214

Grandison A, Ford GD, Millard D, Owen AJ (1984) Chemical composition and coagulating properties of renneted milks from cows during early lactation. J Dairy Res 51, 407416

Grandison AS, Manning DJ, Thomson DJ, Anderson $M$ (1985) Chemical composition, rennet coagulation properties and flavour of milks from cows grazing ryegrass or white clover. J Dairy Res 52, 33-39

Hoden A, Coulon JB, Dulphy JP, Quillet JP (1984) Influence des principaux régimes alimentaires sur les taux butyreux et protéique du lait. In : La composition chimique du lait et ses incidences technologiques. Journées INRA ENSAR INAPG, septembre 1984

Hoden A, Garel JP, Coulon JB, Laurent F (1991) Influence de quelques facteurs zootechniques sur l'aptitude fromagère du lait de vache. Synthèse de résultats expérimentaux obtenus à l'INRA. In : Journées sur la qualité des laits à la production et aptitude fromagère. Colloque INRA-ENSA, Rennes, 23-24 janvier

INRA (1988) Alimentation des bovins, ovins et caprins ( $R$ Jarridge, ed). INRA Publications, route de Saint-Cyr, 78000 Versailles

Journet M, Rémond B (1980) Influence de l'alimentation et de la saison sur les fractions azotées du lait de vache. Lait 60, 140-159

Laurent F, Gardeur JN (1989) Effet de la nature et du niveau d'apport en aliment concentré sur les performances de vaches laitières recevant une ration à base d'ensilage de maïs. Ann Zoolech 38, 247-258

Laurent F, Coomans D, Gardeur JN, Vignon B (1992) Composition azotée et caractéristiques technologiques du lait de vache en relation avec la nature et le niveau d'apport de l'aliment concentré. Lait 72, $175-183$

Le Doré A, Rémond B, Grappin R, Jeunet R, Journet $M$ (1986) Teneurs du lait de vache en ses principales fractions azotées et en matière grasses : effets de quelques caractéristiques des animaux et de leur alimentation. Bull Tech CRZV Theix $63,13-20$

Maubois JL, Ricordeau G, Mocquot G (1970) Étude des rendements en fromagerie de camembert et de saint-paulin. Lait 50, 351-373 
Mocquot G, Ricordeau G, Auriol P (1963) Estimation du rendement en fromage gruyère de comté en fonction de la richesse du lait de chaudière. Ann Zootech 12, 53-66

$\mathrm{Ng}$ Kwai Hang KF, Hayes JF, Moxley JE, Monardes HG (1982) Environmental influences on protein content and composition of bovine milk. J Dairy Sci 65, 1993-1998

Pflimlin A, Morhain B (1988) Comparaison de deux systèmes d'alimentation à deux niveaux de concentré sur cinq années consécutives. La Bouzule 1975-1980. In : Stratégies de complémentation des vaches laitières. ITEB, 149, rue de Bercy, 75595 Paris cedex $12,21-24$

Rémond B (1985) Influence de l'alimentation sur la composition du lait de vache. 2. Taux protéique : facteurs généraux. Bull Tech CRZV Theix 62, 53-67
Rémond B, Journet M (1987) Effet de l'alimentation et de la saison sur la composition du lait. In : Le Lait, matière première de lindustrie laitière. INRA Publications, CNRA, route de Saint-Cyr, 78000 Versailles, 171-186

Rogers GL, Stewart JA (1982) The effect of some nutritional and non-nutritional factors on milk protein concentration and yield. Aust J Dairy Technol 37, 26-32

Sutton JD (1989) Altering milk composition by feeding. J Dairy Sci 72, 2801-2813

Vertes C, Hoden A, Gallard Y (1989) Effet du niveau d'alimentation sur la composition chimique et la qualité fromagère du lait de vaches Holstein et Normandes. INRA Prod Anim 2, 89-96

Vertes C, Hoden A (1989) Qualité fromagère des laits de vache en fonction des régimes à base d'herbe. Lait 69, 197-209 